\title{
Amplification of elastic waves due to a point source in the presence of complex surface topography
}

\author{
António Tadeu *, Paulo Santos, Julieta António \\ Department of Civil Engineering, Faculty of Science and Technology, University of Coimbra, Polo II, Pinhal de Marrocos, 3030-290 \\ Coimbra, Portugal
}

Received 23 May 2000; accepted 27 June 2001

\begin{abstract}
This paper first presents the amplification or de-amplification in the three-dimensional wave scattering generated by the half-space when subjected to a dilatational point load placed at some point in the medium, when the free surface is changed to simulate a two-dimensional ridge or a valley deformation. This model is then extended to include the presence of a long, circular, buried cavity. The solution is formulated using the boundary elements method for a wide range of frequencies and spatially harmonic line loads, which are subsequently used to obtain time series by means of (fast) inverse Fourier transforms into space-time.

Our simulation results indicate larger wave amplification and de-amplification in the time and frequency domains when the topographic surface includes a smooth ridge and canyon deformations. The presence of a buried inclusion further complicates the wave field displacement pattern, including additional reflections. (c) 2001 Elsevier Science Ltd. All rights reserved.
\end{abstract}

Keywords: Boundary elements; Topographic deformations; Point blast source; Seismic wave amplification

\section{Introduction}

The site effects produced by surface topographical deformations have been reported as amplifications and de-amplifications of the seismic signals, which can be significant over large frequency domains [1-3]. Researchers have long been interested in predicting ground movement in the vicinity of different topographical deformations, developing a variety of analytical and numerical approaches to tackle this problem. Some of the first studies report the use of analytical solutions to study the scattering and diffraction produced by alluvial basins of regular shape [4-9] and the wave scattering caused by cavities [10-13]. Semi-analytical methods have been used to analyse the diffraction of waves by geo-

\footnotetext{
${ }^{*}$ Corresponding author. Tel.: +351-239-797-201; fax: +351239-797-190.

E-mail address: tadeu@dec.uc.pt (A. Tadeu).
}

logical inclusions with arbitrary cross-sections placed in a homogeneous medium [14-16]. Numerical methods, such as finite elements and differences, have been used to determine the response within localized, irregular domains, such as the study of soil structure interaction [1719]. In addition to these methods, other researchers have devised techniques for modelling topographic effects, using representation theorems: the direct boundary element method (BEM) $[20,21]$, the indirect boundary element method (IBEM) [22-26]. Another refinement was the development of combinations of integral representations and discrete wave number expansions of Green's functions [27-29]. Discrete methods have also occasionally been used to model large alluvial basins, but only in plane strain [30]. Finally, hybrid methods, which use a combination of semi-analytical representations to model the exterior domain of the inhomogeneities and finite elements to model its interior domain, have also been used [31]. A detailed review of all the methods mentioned above can be found in Sánchez-Sesma [32]. 
These methods have largely been employed modelling the real dynamic problem as a two-dimensional (2D) solution. However, site observation appears to indicate a higher and more broadband amplification than can be predicted by numerical simulations [3,33]. So efforts have been made to extend the $2 \mathrm{D}$ solution so that it can be applied to more realistic, 3D, geological features. The principal drawback of trying to evaluate the whole scattering wave field that is propagated by $3 \mathrm{D}$ media, prompted by locally placed sources, is the huge cost of performing the numerical calculation in terms of computer effort.

A two-and-a-half-dimensional (2.5D) problem, in which the medium is $2 \mathrm{D}$ and the dynamic source $3 \mathrm{D}$ (such as a point load), simplifies the solution. A sequence of two spatial Fourier transforms, in the direction for which the geometry does not vary, is used to solve this type of problem. The first step in the solution involves solving a series of $2 \mathrm{D}$ problems with different spatial wave numbers, $k_{z}$, and then the inverse Fourier transform, can be utilized to synthesize the $3 \mathrm{D}$ field.

The closed form solution can be known for inclusions such as a circular cylinder because the (2.5D) wave equation can be separated. If the inclusion has an irregular cross-section, the solution becomes much harder. The BEM is probably the method best suited to solving problems of this kind. It is almost certainly the best if the inclusions are buried in an unbounded or half-space elastic medium, with the solution thus having to satisfy the far field conditions. The BEM has recently been applied by Stamos and Beskos [34], to a problem where long lined tunnels, with a uniform cross-section, were buried in a half-space. These authors described the 3D dynamic response to plane harmonic waves, propagated in random directions, by treating it as a $2 \mathrm{D}$ problem.

Pedersen et al. [35] used the IBEM to analyse the 3D seismic response of 2D topographic features to plane waves, employing the Green's functions for a harmonic point force moving along the axis of the topography in a full space. These authors present the results for studying the scattering in the frequency and time domains when topographical deformations with simple geometry, such as a semi-circular canyon or a semi-circular ridge, were subjected to incident plane waves. The required numerical equations were derived assuming that the surface topography was divided into a number of segments, each with a constant force distribution. The number of segments was variable with frequency, allowing the existence of five segments per wavelength.

The present work analyses the 3D seismic response of a $2 \mathrm{D}$ smooth canyon or smooth ridge. The medium is subjected to a dilatational point load and the wave field in the zone around the surface deformations is evaluated using the BEM. The surface is discretized along a finite length with varying size elements. The changes caused to the scattered field if a buried cylindrical cavity is present were also studied. In these problems, the solution at each frequency is given in terms of waves with varying wave number, $k_{z}$, where $z$ is the direction in which the geometry does not vary. The result is then Fourier transformed into the spatial domain.

The discrete form of the wave number transform is found by means of the following procedure: an infinite number of virtual point sources are assumed to be spaced at equal intervals along the $z$-axis (the distance between them must be large enough to prevent spatial contamination [36]); complex frequencies, which move down the frequency axis in the complex plane, are used for the analyses to remove any singularities on or near the axis, and so the fictitious sources in the vicinity have only a very small influence [37].

This paper describes, first, the formulation and validation of the BEM through its application to a cylindrical circular cavity, buried in an infinite homogeneous medium and subjected to a dilatational point load, for which the closed form solution is known. The BEM model is then applied to a flat half-space subjected to a line blast load, for which the closed form solution is also known (Green's function [28]). This model is next used for simulation analyses to investigate the three-dimensional wave propagation alteration in the vicinity of a flat half-space when a smooth canyon or a smooth ridge is introduced. The amplifications and de-amplifications of the seismic signals are again studied when a long buried cavity with a circular cross-section is placed below the topographical deformation. The quantitative study of the 3D effects of scattering has been achieved by obtaining results in the frequency and time domains, paying particular heed to the different apparent wave velocities along the $z$-axis.

\section{Problem formulation for $\mathbf{2 . 5 D}$}

The incident field generated by a harmonic dilatational point source in an elastic uniform medium can be expressed by means of the classic dilatational potential $\phi$

$$
\phi_{\text {inc }}=\frac{A \exp \left(\mathrm{i} \frac{\omega}{\alpha}\left(\alpha t-\sqrt{\left(x-x_{0}\right)^{2}+\left(y-y_{0}\right)^{2}+z^{2}}\right)\right)}{\sqrt{\left(x-x_{0}\right)^{2}+\left(y-y_{0}\right)^{2}+z^{2}}}
$$

in which $\omega$ is the oscillating frequency, $\left(x_{0}, y_{0}, 0\right)$ is the position of the load, the subscript inc denotes the incident field, $A$ is the wave amplitude, $\alpha$ is the compressional wave velocity of the medium, and $\mathrm{i}=\sqrt{-1}$. Fourier-transforming Eq. (1) in the $z$ direction, and using the effective wave numbers, $k_{\alpha}=\sqrt{\left(\omega^{2} / \alpha^{2}\right)-k_{z}^{2}}$ 
with $\operatorname{Im} k_{\alpha}<0$, where $k_{z}$ is the axial wave number, one obtains

$\hat{\phi}_{\mathrm{inc}}\left(\omega, x, y, k_{z}\right)=\frac{-\mathrm{i} A}{2} H_{0}^{(2)}\left(k_{\alpha} \sqrt{\left(x-x_{0}\right)^{2}+\left(y-y_{0}\right)^{2}}\right)$

in which the $H_{n}^{(2)}(\cdots)$ are second Hankel functions of order $n$.

If one assumes the existence of an infinite set of evenly spaced sources along the $z$ direction, the former incident field may be written as

$\phi_{\text {inc }}(\omega, x, y, z)=\frac{2 \pi}{L} \sum_{m=-\infty}^{\infty} \hat{\phi}_{\text {inc }}\left(\omega, x, y, k_{z m}\right) \mathrm{e}^{-\mathrm{i} k_{z m} z}$

where $L$ is the spatial source interval, and $k_{z m}=(2 \pi /$ $L) m$. Thus, the three-dimensional wave field may be obtained as the wave irradiated by a sum of harmonic (steady state) line loads whose amplitude varies sinusoidally in the third dimension. This sum converges and can be approximated by a finite number of terms.

\section{Boundary element formulation}

\subsection{Cylindrical irregular cavity}

The BEM is used to compute the 3D field generated by a cylindrical irregular cavity when illuminated by spatially sinusoidal harmonic line loads, defined by Eq. (2) (see Fig. 1). The required BEM equations are widely known (see Refs. [38,39]), and only a brief explanation of the method to be applied in the frequency domain, is presented. The boundary integral equations can be constructed using the dynamic reciprocal theorem, through which, in the absence of distributed loads and in the presence of virtual point loads, $\delta\left(x-x_{0}\right)$, the following equation is defined,

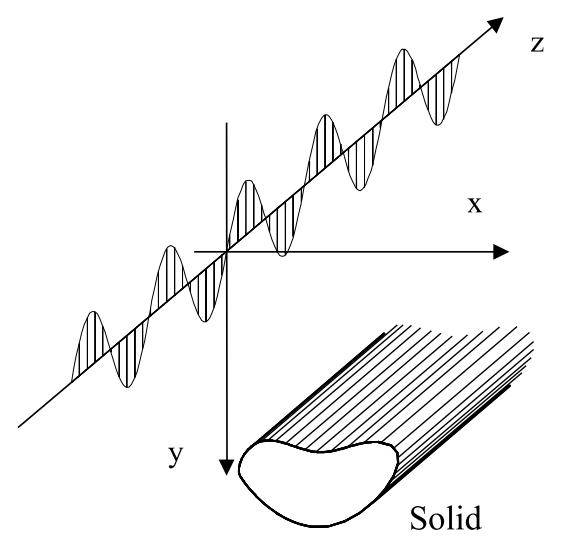

Fig. 1. Problem geometry.

$$
\begin{aligned}
c_{i j} u_{j}\left(x_{0}, \omega\right)= & \int_{C} t_{i}(x, v, \omega) G_{i j}\left(x, x_{0}, \omega\right) \mathrm{d} s \\
& -\int_{C} H_{i j}\left(x, v, x_{0}, \omega\right) u_{j}(x, \omega) \mathrm{d} s
\end{aligned}
$$

In this equation $i, j=1,2$ stands for the normal and tangential directions relative to the cavity surface, respectively, while $i, j=3$ refers to the $z$ direction. $G_{i j}\left(x, x_{0}, \omega\right)$ and $H_{i j}\left(x, v, x_{0}, \omega\right)$ are the displacements and tractions in the direction $j$ at $x$ (on the boundary $C$ ) as a result of a unit point force in the direction $i$ at $x_{0}$ (the source). The vector $v$ is the unit outward normal at the boundary, while $c_{i j}$ is a constant, depending on the local geometry of the boundary. For a smooth boundary, $c_{i j}$ is equal to $\delta_{i j} / 2$, where $\delta_{i j}$ is the Kronecker's delta.

Expressions for the tensions can be computed from the 2.5D Green's functions, by taking partial derivatives to deduce the strains and then applying Hooke's law to find the stresses. The displacement and stress functions, in Cartesian co-ordinates, which apply to the present problem, are listed in Appendix A. These stress fields are conveniently transformed into the normal, tangential and $z$ local co-ordinate system at each element by equilibrium relations. The boundary conditions at the boundary of a cavity prescribe null tractions, leading to the simplified form of Eq. (4),

$c_{i j} u_{j}\left(x_{0}, \omega\right)=-\int_{C} H_{i j}\left(x, v, x_{0}, \omega\right) u_{j}(x, \omega) \mathrm{d} s$

The evaluation of this integral for an arbitrary crosssection requires the discretization of both the boundary and boundary values. If $N$ boundary elements are used and the nodal displacements are assumed to be constant within each element, and equal to the value at the associated nodal point, Eq. (5) changes to

$c_{i j} u_{j}^{k}=-\sum_{n=1}^{N} H_{i j}^{k n} u_{j}^{n}$

In this equation, $k$ is the element number at the point where the virtual load is applied, $u_{j}^{n}$ is the boundary values in element $n$, and $H_{i j}^{k n}$ is the element integrals

$H_{i j}^{k n}=\int_{C_{n}} H_{i j}\left(x_{n}, v_{n} x_{k}, \omega\right) \mathrm{d} s$

in which $v_{n}$ is the unit outward normal for $n$th boundary segment $C_{n}$.

By successively applying the virtual load to each node on the boundary, a system of linear equations relating nodal forces and nodal displacements is obtained, and these can be solved for the nodal displacements.

If an incident wave strikes the cavity, Eq. (5) changes to 
$c_{i j} u_{j}\left(x_{0}, \omega\right)=-\int_{C} H_{i j}\left(x, v, x_{0}, \omega\right) u_{j}(x, \omega) \mathrm{d} s+u_{j}^{\mathrm{inc}}\left(x_{0}, \omega\right)$

In this equation the incident field is obtained by taking partial derivatives of Eq. (2) to deduce displacements along $x, y$ and $z$, and then applying equilibrium relations to obtain the normal and tangential displacements along the boundary.

If the displacements and tractions are allowed to vary linearly within the boundary elements, the required integrals of Eq. (7) change to

$H_{i j}^{k n}=\int_{C_{n}} \phi H_{i j}\left(x_{n}, v_{n}, x_{k}, \omega\right) \mathrm{d} s$

where $\phi$ are the linear interpolation functions.

Within a boundary element, the displacement and stress variations are defined in terms of the nodal values. The traction discontinuity at the corner between two boundary elements is handled by moving the nodes that would meet at the corner, so that each is inside an element [40], leading to discontinuous boundary elements.

This gives a system of equations that can be solved for the nodal displacements, after combining the integral equations and subjecting them to the continuity conditions at the interface between the two media (null tractions). The required integrations in Eq. (9) are performed in closed form when the element to be integrated is the loaded element [41,42], while numerical integration, using a Gaussian quadrature scheme, applies when the element to be integrated is not the loaded one.

The BEM algorithm was implemented and validated by calculating the response of a cylindrical circular cavity, placed in a homogeneous elastic medium, when subjected to a dilatational point load, for which the solution is known in closed form. Details of this validation can be found in Ref. [43] and they are not presented here for reasons of brevity.

\subsection{Surface topography}

The BEM model developed for the cylindrical cavity can be used to calculate the response generated by a free flat surface (half-space). In fact, the same equations apply over the boundary elements required to simulate the free surface. The geometrical damping together with the use of complex frequencies makes the full discretization of the infinite surface unnecessary. If solutions are required in the time domain, the contribution to the response that arrives behind the time window $T=2 \pi / \Delta \omega$ (where $\Delta \omega$ is the frequency step of the analysis) does not need to be taken into account. Hence, the free surface needs to be discretized up to a spatial distance $\left(L_{\text {dist }}\right)$ from the receivers, given by $L_{\text {dist }}=\alpha T$. This leads to a total discretized surface with a length $2 L_{\text {dist }}+2 a$, where $2 a$ is the length of the segment occupied by the receivers.

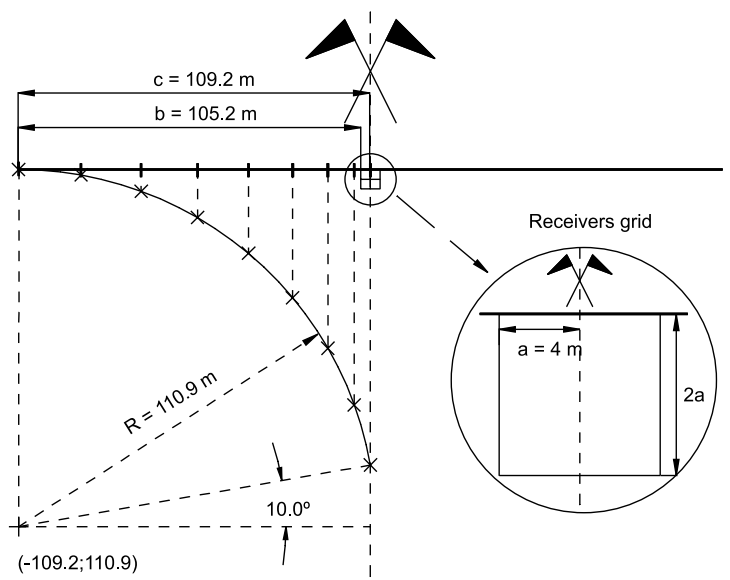

Fig. 2. Boundary elements distribution $(f=100 \mathrm{~Hz} ; \Delta f=40$ $\mathrm{Hz})$.

Many schemes for distributing the boundary elements along the discretized surface were tested to study how varying the size of boundary elements affects the accuracy of the response. A better performance was found when smaller elements were placed in the vicinity of the receivers. The authors of this paper suggest placing differently sized boundary elements along the discretized surface, with the shorter elements being used nearer to the centre of the surface discretization. The scheme used in this work to define the size of the boundary elements is illustrated in Fig. 2. The method comprises a geometrical construction in which the ratio between the wavelength of the dilatational waves and that of the boundary elements is first defined, and then used to divide an auxiliary circular arc into equal segments. These segments are projected vertically to define the boundary elements on the topographic surface. The radius of the circular arc required $(R)$ is greater than $\left(2 L_{\text {dist }}+2 a\right) / 2$ and it is placed at the boundary discretization end, tangent to the topographic surface, avoiding the existence of unduly small boundary elements. In this work $R$ is assumed to be $\left[\left(2 L_{\text {dist }}+2 a\right) / 2\right] / \cos 10^{\circ}$.

The BEM algorithm was implemented and validated by applying it to a flat half-space, subjected to a dilatational line load (Fig. 3a), for which the solution is known in closed form [28].

Fig. 3 illustrates the computation of the vertical displacement field over a fine vertical grid plane, which is perpendicular to the $z$-axis, when a $100 \mathrm{~Hz}$ pressure line load is excited. The response given by the closed form solution is shown in Fig. 3b. Fig. 3 also displays the difference, or error, given by the BEM when 122 boundary elements are used and the ratio between the wavelength of the dilatational waves and the boundary elements is 10 . Fig. $3 \mathrm{c}$ shows the error in the case where boundary elements are spaced at equal distances from 


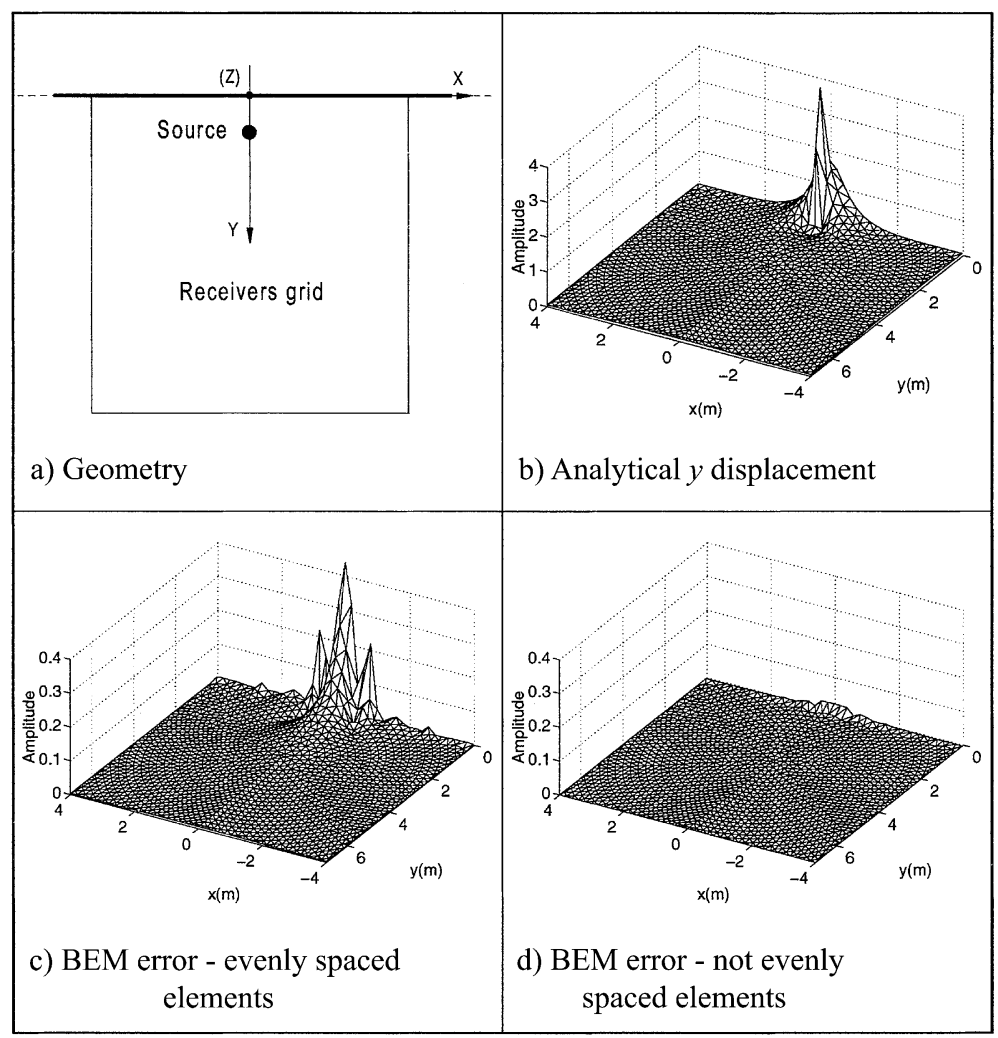

Fig. 3. Half-space problem validation.

one another along the surface boundary, while Fig. 3d gives the error when the length of the boundary elements changes in accordance with the method illustrated in Fig. 2. The accuracy of the BEM is enhanced, as these figures show, when shorter elements are used towards the centre of the surface discretization, at no extra computer cost.

\section{Transformation from frequency to time domain - use of a Ricker wavelet}

The responses in the time-space domains are obtained by means of inverse Fourier transforms over both the frequency and wave number domains. The dynamic source is assumed to have a Ricker wavelet temporal variation, and this decays rapidly in both the time and frequency domains, reducing computational effort, and allowing the computed synthetic waveforms to be interpreted more easily.

The Ricker wavelet function in the frequency domain is given by

$U(\omega)=A\left[2 \sqrt{\pi} t_{0} \mathrm{e}^{-\mathrm{i} \omega t_{s}}\right] \Omega^{2} \mathrm{e}^{-\Omega^{2}}$

where $A$ is the amplitude, $\Omega=\omega t_{0} / 2, t_{s}$ denotes the time when the maximum occurs, while $\pi t_{0}$ is the characteristic (dominant) period of the wavelet.
The required Fourier transformations are obtained by discrete summations over wave numbers and frequencies, which is mathematically the same as adding periodic sources at spatial intervals $L=2 \pi / \Delta k_{z}$ (in the $z$ axis), and temporal intervals $T=2 \pi / \Delta \omega$, with $\Delta k_{z}$ and $\Delta \omega$ being the wave number and frequency steps, respectively [36]. The spatial separation $L$ must be large enough to ensure that there is no contamination of the response by the periodic sources. In addition, the contribution to the response by the fictitious sources must occur at times later than $T$. Analyses are therefore performed using complex frequencies, with a small imaginary part of the form $\omega_{c}=\omega-\mathrm{i} \eta$ (with $\eta=0.7 \Delta \omega$ ), and so the influence of the neighbouring fictitious sources is practically non-existent [37]. In the time domain, this shift is later taken into account by applying an exponential window $\mathrm{e}^{\eta t}$ to the response (see Ref. [44]).

\section{Numerical applications}

The BEM model is first used to calculate the threedimensional wave field generated by $2 \mathrm{D}$ smooth topographical deformations subjected to a dilatational point load. The topographical surface is modelled with a number of boundary elements, defined according to the 


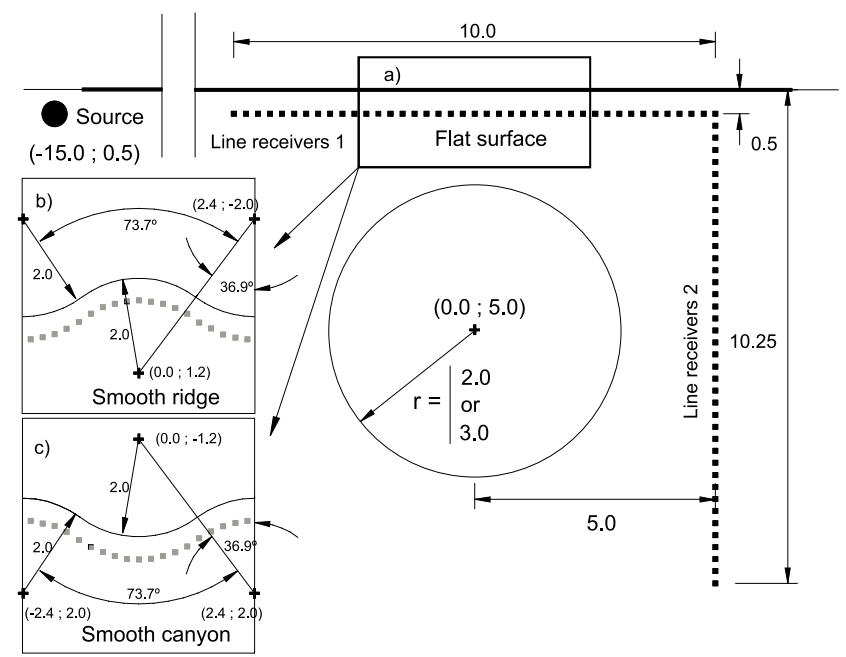

Fig. 4. Geometry for numerical applications.

excitation frequency of the harmonic source, and in such a way that the free surface can be discretized along a distance that is sufficient to fully reproduce the responses at the receivers in the time window considered $(T=$ $1 / \Delta f)$. Simulation analyses are performed to study the alteration of the wave scattering generated by the halfspace, when the free surface is changed to simulate a smooth ridge or a valley, defined with circular arcs of constant radius, $2 \mathrm{~m}$ (see Fig. 4).

Next, the BEM model is further extended to include the presence of a long, circular, buried cavity. The cavity is placed below the topographical deformation, with its axis positioned at $x=0 \mathrm{~m}$ and $y=5 \mathrm{~m}$. Computations are performed for two sizes of circular inclusions with radii $R=2 \mathrm{~m}$ and $R=3 \mathrm{~m}$.

The shear wave velocity $(\beta=2656 \mathrm{~m} / \mathrm{s})$, the dilatational wave velocity $(\alpha=4208 \mathrm{~m} / \mathrm{s})$ and density $(\rho=$ $2140 \mathrm{Kg} / \mathrm{m}^{3}$ ) of the elastic medium remain constant in all the analyses. Computations are performed in the frequency range $(40-1280 \mathrm{~Hz})$, with a frequency increment of $40.0 \mathrm{~Hz}$, which determines the total duration $(T=25.0 \mathrm{~ms})$ of the analysis in the time domain. The source time dependence is a Ricker wavelet with a characteristic frequency of $450 \mathrm{~Hz}$.

The propagating wave field is computed at 80 evenly spaced $(0.25 \mathrm{~m})$ receivers, distributed along two lines: one is placed horizontally at $0.5 \mathrm{~m}$ below the surface (line 1), while the second is placed vertically at $x=5.0 \mathrm{~m}$ (line 2). The horizontal line of receivers could be chosen to coincide with the nodal boundary elements. However, this procedure has been avoided for two reasons. First, because the surface is not equally discretized along the boundary, and second, the authors are trying to simulate the placement of the geophones at some depth below the surface to avoid (in practice) unwanted noise.
At time $t=0$, a point source placed $0.5 \mathrm{~m}$ below the free surface, at $x=-15 \mathrm{~m}$ and $z=0 \mathrm{~m}$, creates a spherical dilatational pulse propagating away from it.

The free topographical surface and the cavities are modelled with a number of boundary elements that changes with the excitation frequency of the harmonic load. Many simulations were performed to study how varying the size of boundary elements affects the accuracy of the response. The ratio between the wavelength of the incident waves and the length of the boundary elements is kept to a minimum of 10 . In all cases, however, the number of the boundary elements used to model each surface is never less than 40 .

Responses are calculated following waves with different apparent wave velocities along the $z$-axis. This apparent wave velocity $(c)$ results from waves arriving at the $z$-axis with a path inclination given by $\arccos (v / c)$, where $v$ is the true wave velocity (see Fig. 5). $k_{z}$ is taken to be $\omega / c$ in the BEM equations. Selected results are presented for simulations with three apparent velocities (c): infinite $c, c=4208 \mathrm{~m} / \mathrm{s}$ and $c=2656 \mathrm{~m} / \mathrm{s}$. Infinite $c$ corresponds to waves arriving at the receivers with a $90^{\circ}$ inclination in relation to the $z$-axis, which can be seen as

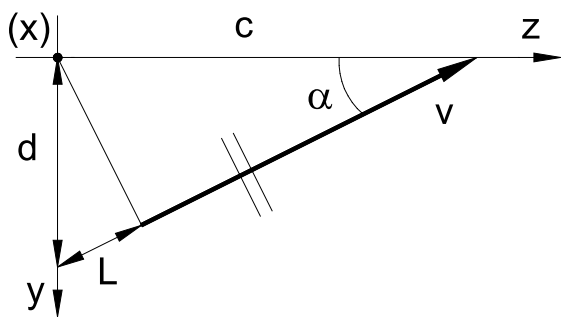

Fig. 5. Apparent wave velocity. 
a pure $2 \mathrm{D}$ problem where the source is linear. As the path inclination ranges from $90^{\circ}$ to $0^{\circ}$, there is a lower bound value for $c$ that corresponds to the slowest wave velocities (Rayleigh waves). Below this value, there are inhomogeneous waves which decay very quickly with decreasing values of $c$.

\section{1. $2 D$ topographical deformations free of any buried inclusion}

Fig. 6 displays the synthetic seismograms in the time and frequency domains for the horizontal $(x)$ displace- ment, recorded at receivers placed along line 1 , for the three topographical surfaces. Solid lines placed on the limits of the topographical deformations and on their inflection points (locating the change of curvature from convex to concave) are added to facilitate interpretation of the results.

Fig. 6a shows the response obtained for infinite $c$. When the surface is flat, the first set of pulses recorded at the receivers corresponds to the incident $P$ field and $P$ waves reflected from the surface, while second arrivals are $S$ mode converted waves resulting from the $P$ wave incidence on the surface. The $S$ waves are followed by (a)
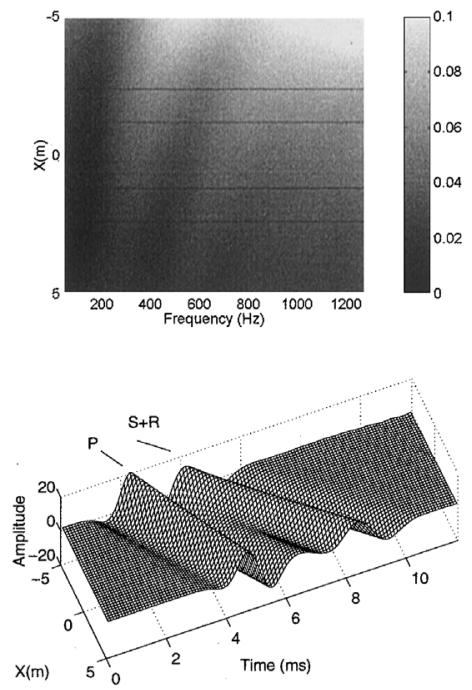

(b)
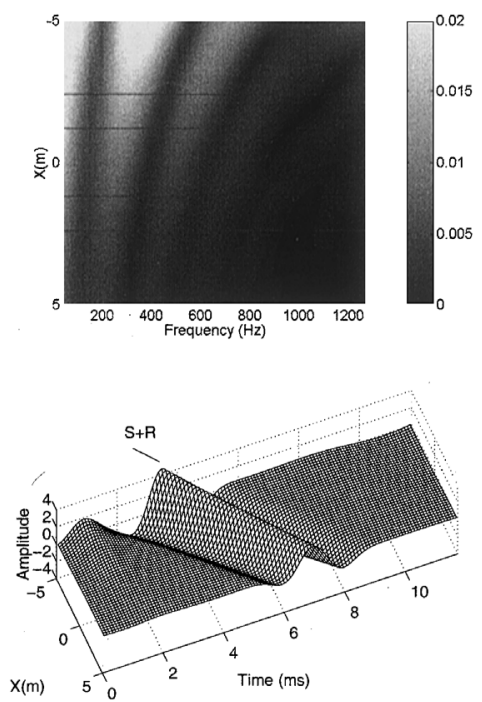

Smooth ridge
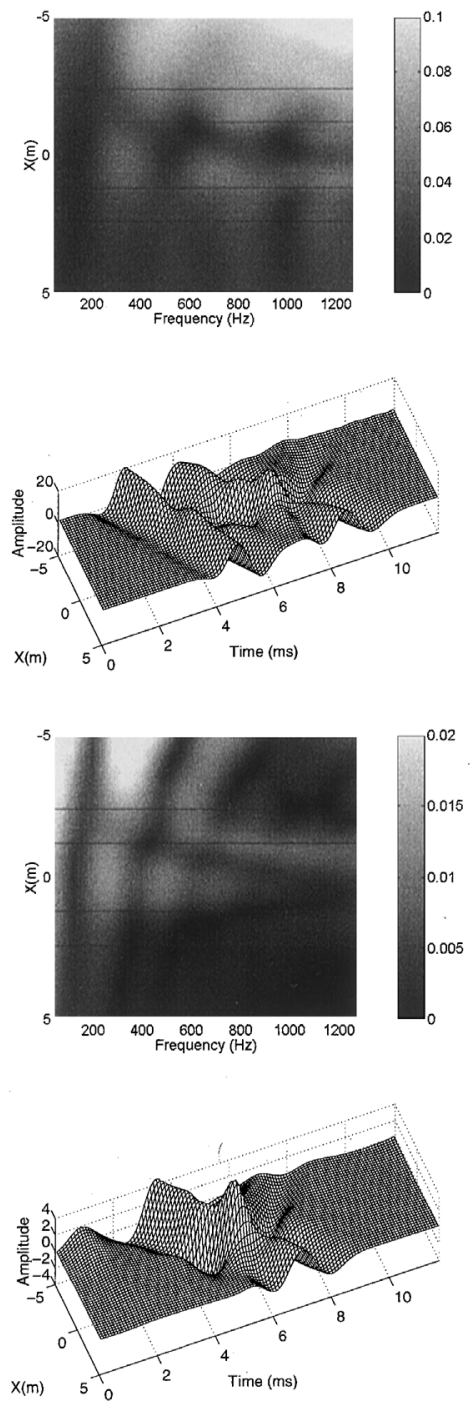

\section{Smooth canyon}
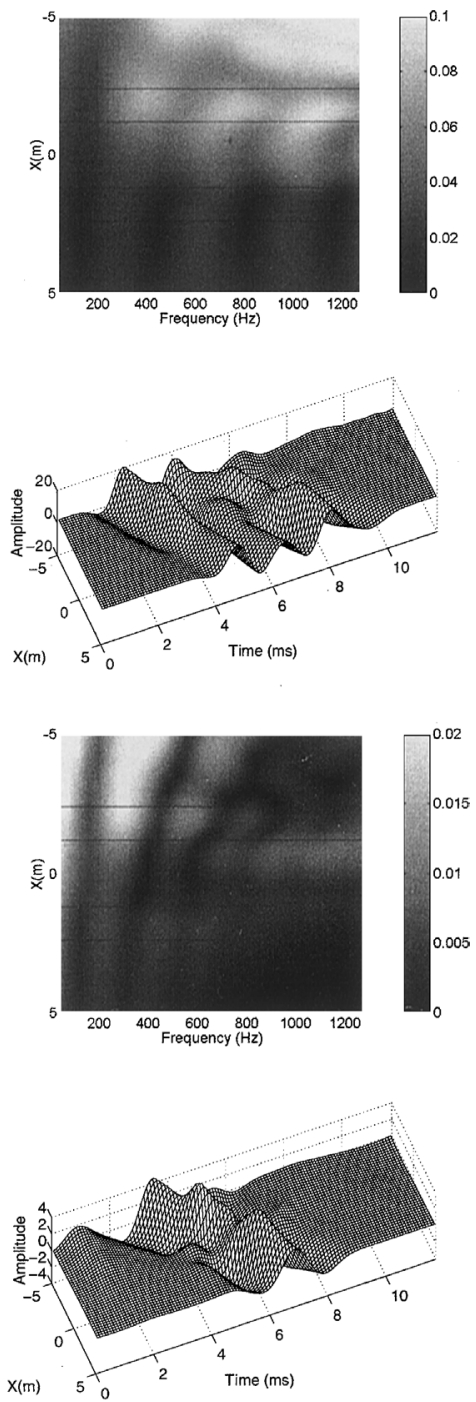

Fig. 6. Total horizontal displacement time and frequency responses recorded at the receivers placed along line 1: (a) Infinite $c$, (b) $c=4208 \mathrm{~m} / \mathrm{s}$ and (c) $c=2656 \mathrm{~m} / \mathrm{s}$. 
(c)
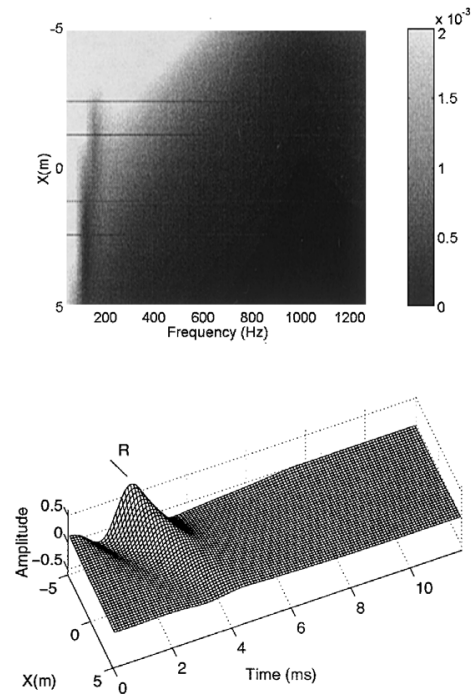
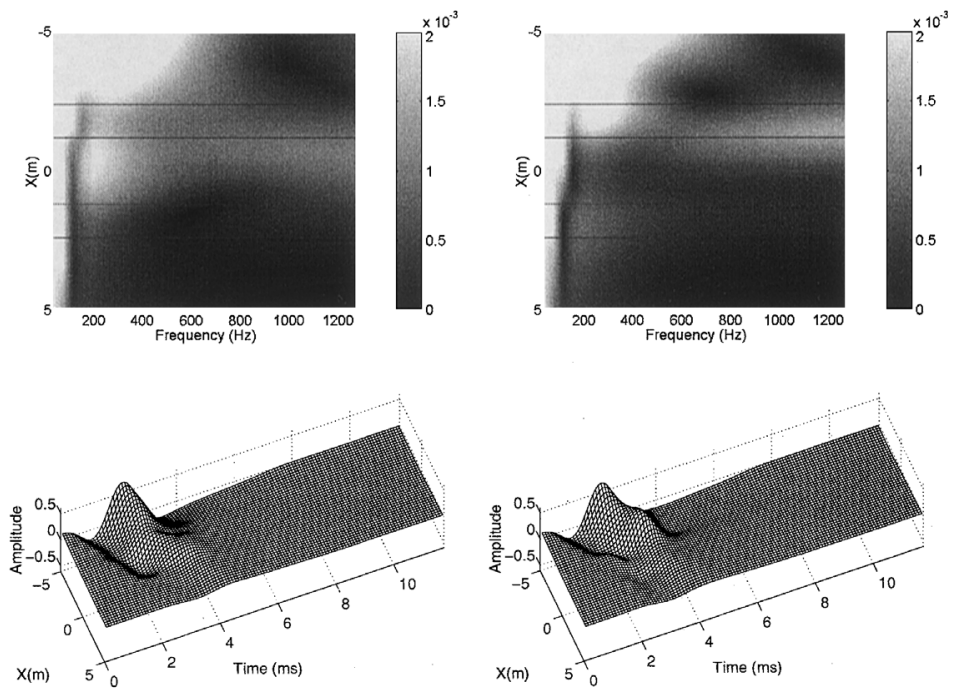

Fig. 6 (continued) the guided waves $(R)$, but they do not fully separate as two pulses, given the small difference in velocities. Tags are added in these plots indicating the arrivals of the various pulses. It can easily be demonstrated that the arrival times obtained for the different pulses in these plots are consistent with the predictions given by ray acoustics.

When the topographical surface is irregular, the responses show an increased interference, which grows with frequency. In the case of the smooth ridge, the interference is caused mainly by reverberations within the concave part of the surface. A more pronounced frequency wave field difference amplitude response is observable at receivers placed below the central part of the deformation, agreeing with this interpretation. It can be further observed that the synthetic seismograms show signal amplification and de-amplification at those same receivers. In the case of the smooth canyon, amplification of the signal occurs, but here it is for receivers close to the extreme of the deformation, again within the concave parts of the free surface. As expected, higher responses occur at the edge nearer to the dynamic source. The presence of the ridge and the canyon causes a "shadow" after the deformations, which is more pronounced in the case of a canyon.

As the apparent velocity decreases the arrival times of the different pulses decrease (see Fig. 6b and c). A pulse with an arrival time $\tau$ in these plots relates to waves that travel from the source to a reflector and then to the receiver, along a constant ray path inclination in relation to the $z$-axis, $\arccos (v / c)$. The travel distance $(L)$ in this domain corresponds to the projection of the initial vertical path $(d)$ relative to the inclined path,
$L=d \sin [\arccos (v / c)]$. In this way, when the apparent velocity reaches the velocity of the $P$ waves (see Fig. 6b), the pulses travelling at this velocity arrive at the various receivers at $t=0 \mathrm{~ms}$, and only the waves travelling at lower velocity, the $S$ and guided waves, survive in these time plots. Furthermore, when the apparent velocity equals $c=2656 \mathrm{~m} / \mathrm{s}$, only the guided waves (Rayleigh waves) survive.

Analysis of the results shows that the main features of the wave patterns described for $c=\infty \mathrm{m} / \mathrm{s}$ are maintained as the apparent velocity decreases. The concave parts of the surface are still defining the position of the amplification and de-amplification of the former signals obtained for the flat half-space. However, the wave field interference originating on the topographic deformations becomes less significant as the apparent wave velocity decreases.

Fig. 7 illustrates the vertical displacement at the receivers, again placed along line 1 for infinite $c$. The $P$ pulses decrease in importance, because their displacement is mostly polarized along the horizontal direction, given the position of both the source and the receivers, placed just below the free surface. The scattered field exhibits wave features similar to those found for the horizontal wave displaced by the amplification of the signal in the vicinity of the concave parts of the topographical surfaces. As the apparent velocity decreases, the interference from the different topographical deformations rapidly loses its importance (not illustrated). The $z$ displacements, null for infinite $c$, denote similar features (not illustrated).

Fig. 8 displays the synthetic seismograms recorded at the receivers placed along line 2 . The time of flight of 
Flat surface

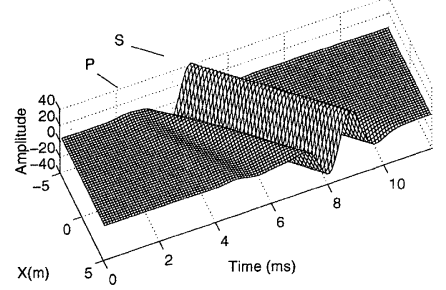

Smooth ridge

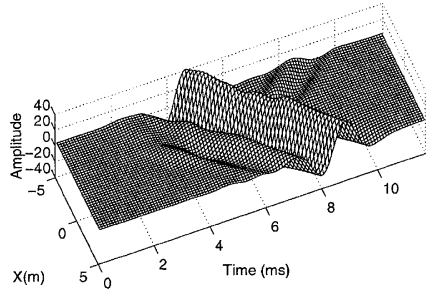

Smooth canyon

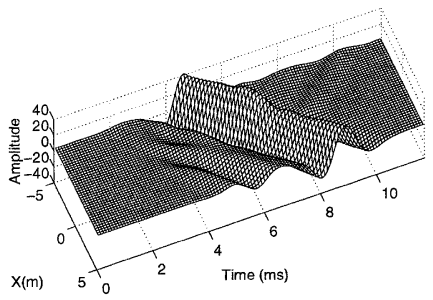

Fig. 7. Total vertical displacement time and frequency responses recorded at the receivers placed along line 1 for infinite $c$.

(a)

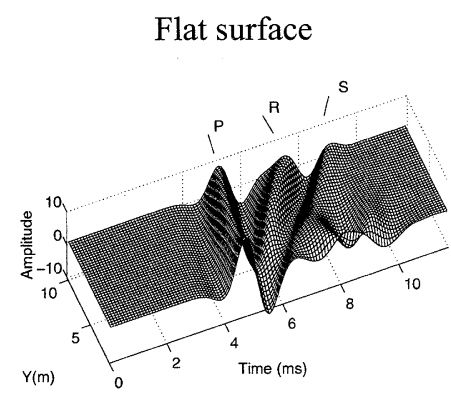

(b)

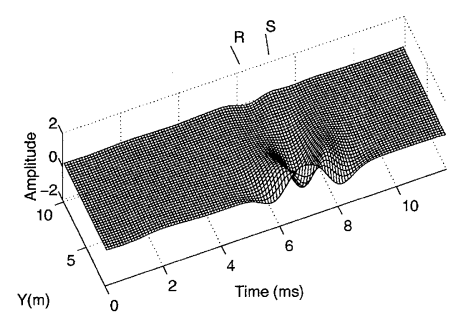

Smooth ridge
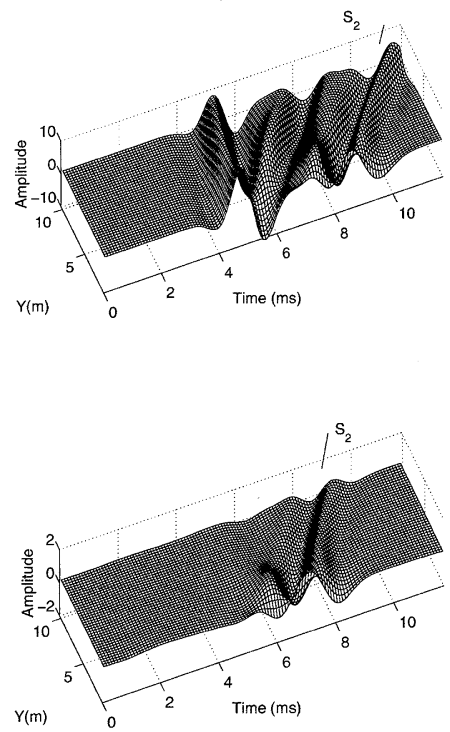

Smooth canyon
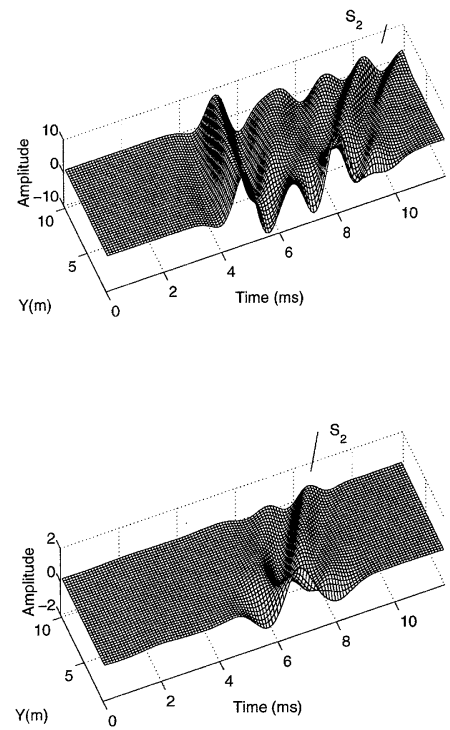

Fig. 8. Total horizontal displacement time responses recorded at the receivers placed along line 2: (a) Infinite $c$ and (b) $c=4208 \mathrm{~m} / \mathrm{s}$.

each of these pulses agrees with the ray analyses. Fig. 8a depicts the horizontal $(x)$ displacement when the apparent velocity is infinite $c$.

The reverberations within the topographical surface deformations leads to more complicated wave patterns, with the presence of additional pulses. The pulse that reflects on the surface topographical deformation as an $S$ pulse is identified as $S_{2}$ in these plots. The pulses directly incident on the canyon deformation are readily reflected into the formation, creating a shadow behind the surface deformation, as the fall in amplitude of the pulses recorded at receivers placed at an intermediate depth indicates. At the receivers placed close to the surface, the pulses resulting from the guided modes dominate the response. The results further show that these guided waves, which decay very rapidly with depth, are more pronounced in the presence of the canyon than in the presence of the ridge. When the apparent velocity is $c=4208 \mathrm{~m} / \mathrm{s}$ (Fig. $8 \mathrm{~b}$ ), the wave field still shows the effect of the shadow phenomenon, but now significantly reduced.

The vertical and $z$ displacements, not illustrated here, exhibit similar wave features.

\subsection{Presence of a buried cavity below $2 D$ topographical deformations}

This section presents the results obtained when a cylindrical circular cavity is placed below the topographical deformations. The medium is again excited by a point source placed $0.5 \mathrm{~m}$ below the free surface, at $x=-15 \mathrm{~m}$ and $z=0 \mathrm{~m}$, creating a spherical pressure 
pulse propagating away from it. The field generated is computed at the same two lines of receivers.

Fig. 9 displays the horizontal $(x)$ displacement in the time and space-frequency domains recorded at the receivers placed at line 1 , for the flat free surface, the smooth ridge and the smooth canyon, in the presence of a cavity with a radius of $R=3 \mathrm{~m}$. Analysis of the results reveals that the presence of this inclusion leads to a more complicated wave field displacement pattern, including additional reflections by the cavity (see Fig. 6). The results continue to exhibit amplification and de-amplification close to the concave part of the surface deformations.
As waves impinge on both the cavity and the free surface they scatter back into the medium as $P$ and $S$ waves (the latter as the result of $P-S$ conversion). The presence of the ridge permits higher order reflections between the surface and the top of the inclusion, sustaining more energy there, for a longer time. This phenomenon gives rise to additional pulses, owing to the interference between these reflections. The receivers placed in the vicinity of the canyon show smaller amplitudes than do the receivers that are further away. This phenomenon is explained by the reflective power of the convex shape of the surface and of the cavity, which enables energy to be reflected to the sides, away from the (a)
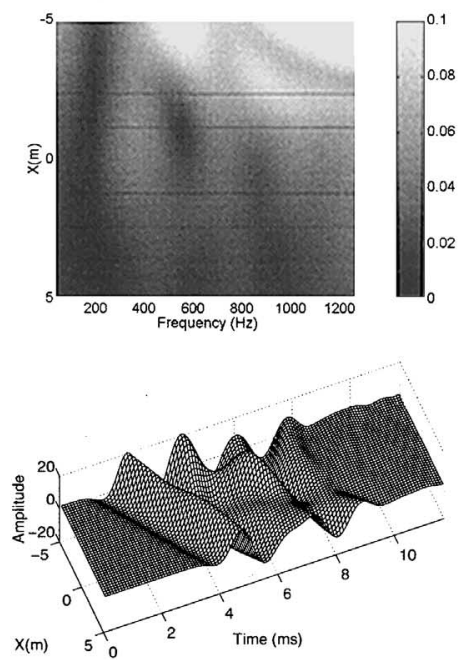

(b)
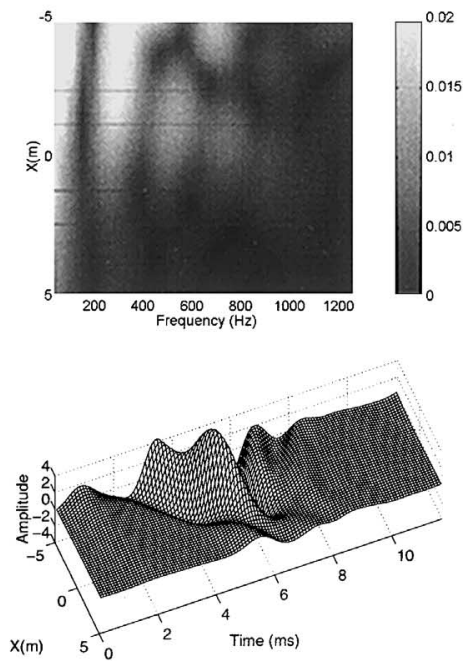

Smooth ridge
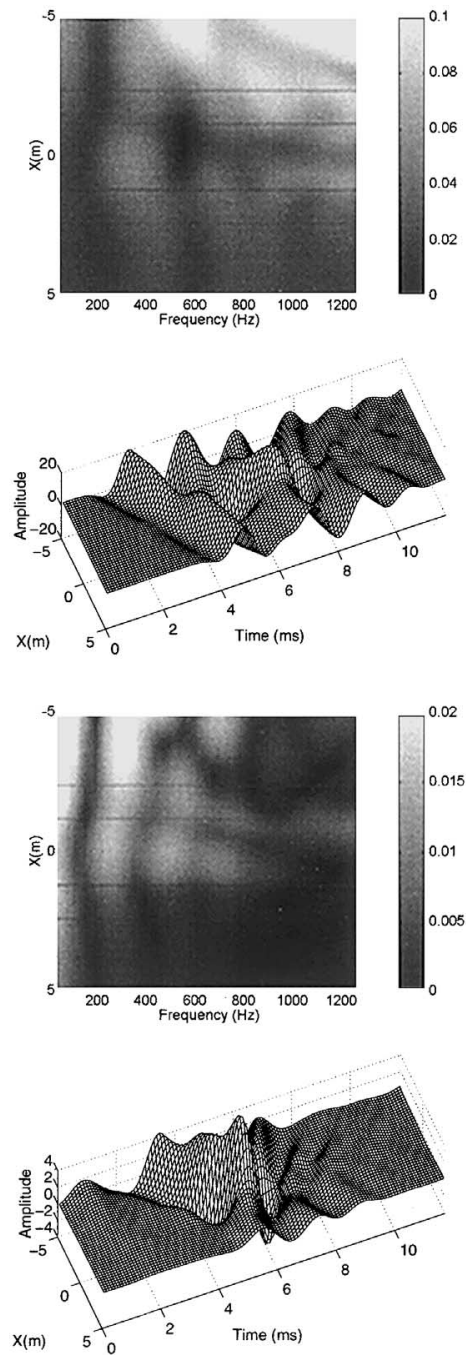

Smooth canyon
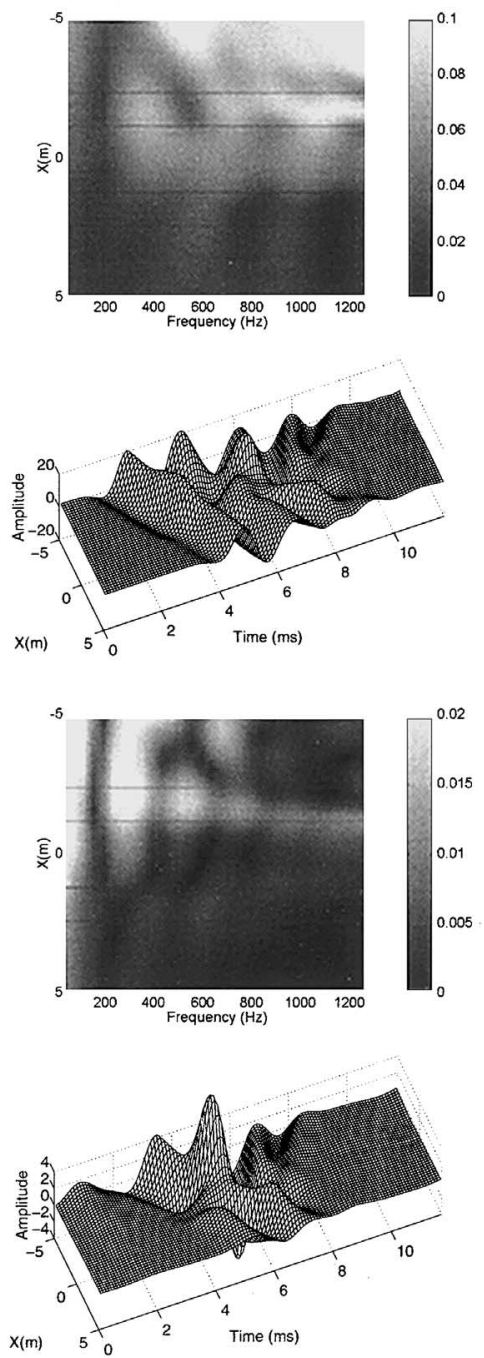

Fig. 9. Total horizontal displacement time and frequency responses recorded at the receivers placed along linel when a cylindrical circular cavity of $R=3 \mathrm{~m}$ is present: (a) Infinite $c$, (b) $c=4208 \mathrm{~m} / \mathrm{s}$ and (c) $c=2656 \mathrm{~m} / \mathrm{s}$. 
(c)
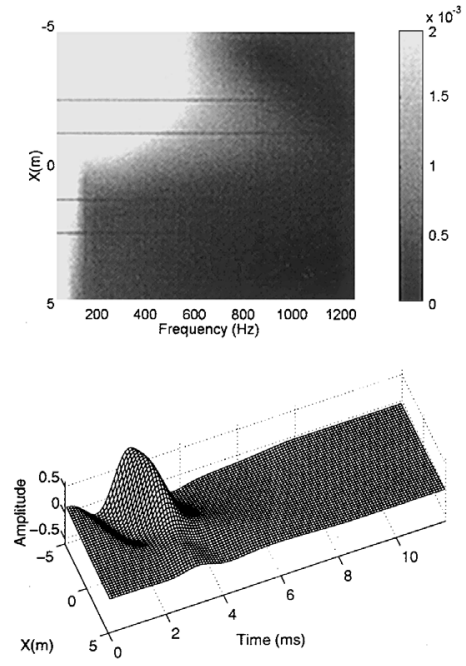
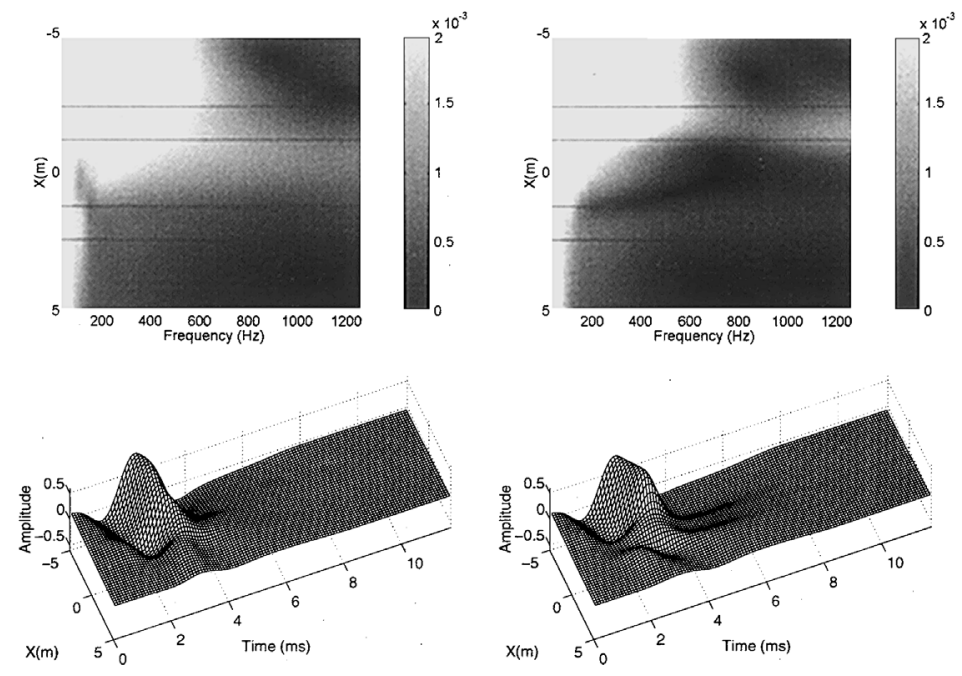

Fig. 9 (continued)

central zone. However, the wave field generated is not symmetric in relation to the central part of the surface deformation, but is more pronounced at the side where the dynamic source is located. The ridge and canyon again produce a shadow behind the deformations, which it is more pronounced in the case of a canyon.

As the apparent velocity decreases, the amplitude of the displacement decreases, and the waves that travel at a velocity higher than the assumed apparent velocity vanish. Our results indicate the existence of signal amplifications and de-amplifications in the vicinity of the concave parts of the deformations (see Fig. 9b), with amplifications bigger than the ones found for the medium with no buried cavity, a homogeneous medium (see Fig. 6b). As found before, the importance of the guided waves decreases as the distance of the receivers from the source increases (see Fig. 9c). It can be further observed, that the guided waves now have higher amplitudes than were observed for them in the homogeneous medium (see Fig. 6c). This is caused by the additional presence of guided waves that travel around the surface cavity.
Fig. 10 illustrates the vertical displacement $(y)$ for the same receivers (infinite $c$ ). It can be seen that the displacement in the vertical direction gives amplitudes higher than those given by displacement in the horizontal direction, especially at the receivers closer to the source. Once again the importance of the $P$ waves decreases. As the frequency increases, the wave signal exhibits amplification patterns in the vicinity of the concave parts of the topographical surfaces similar to the ones found for the horizontal wave displacement (not illustrated). The presence of a ridge allows a longer dynamic event, as explained before. As the apparent velocity decreases, the interference of the different topographical deformations rapidly loses its importance (not illustrated). As for the horizontal displacements, the Rayleigh waves increase their relative importance, owing to the presence of the cavity.

Fig. 11 displays the $z$ displacement at receivers placed again at line 1 , when the apparent velocity is $c=4208$ $\mathrm{m} / \mathrm{s}$. The presence of a buried cavity causes a more pronounced interference. As before, the space-frequency domain maintains higher amplitude scattered responses
Flat surface

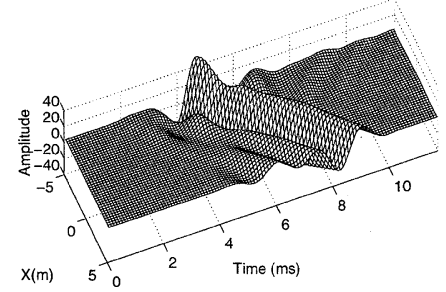

Smooth ridge

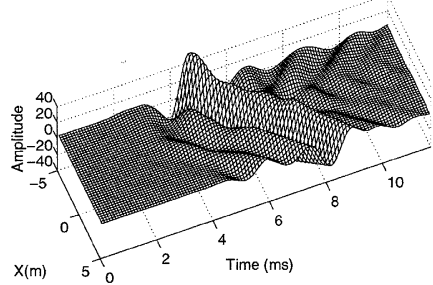

Smooth canyon

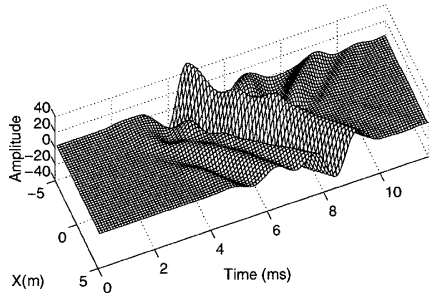

Fig. 10. Total vertical displacement time responses recorded at the receivers placed along line 1 when a cylindrical circular cavity of $R=3 \mathrm{~m}$ is present - infinite $c$. 
Flat surface

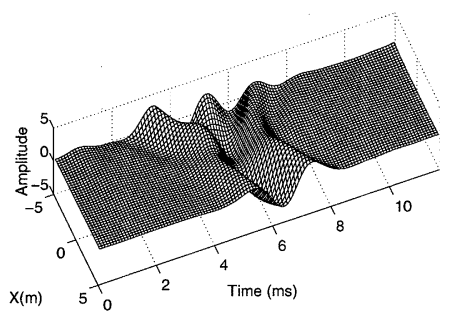

Smooth ridge

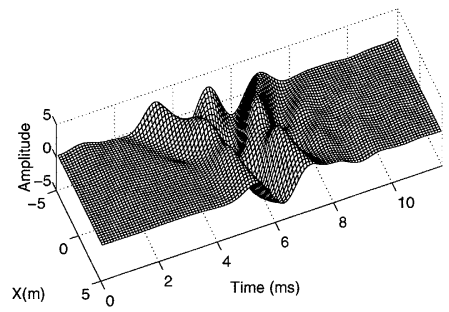

Smooth canyon

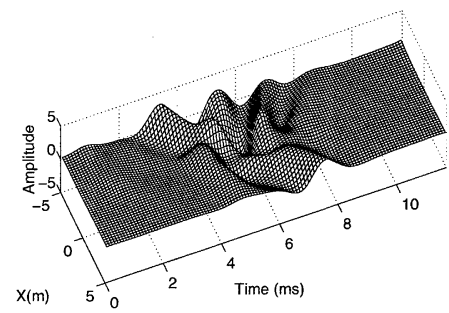

Fig. 11. Total $z$ displacement time responses recorded at the receivers placed along line 1 when a cylindrical circular cavity of $R=3 \mathrm{~m}$ is present for $c=4208 \mathrm{~m} / \mathrm{s}$.

in the vicinity of the concave part of the surface deformations, as the frequency increases (not illustrated).

Fig. 12 illustrates the horizontal response for different apparent wave velocities. When infinite $c$, the first wave arrivals observed correspond to the incident waves followed by wave trains that are directly reflected by the surface and the cavity. The cavity causes a so-called "shadow effect" when illuminated by the first incident $P$ body waves, placing smaller amplitude pulses behind the cavity (see also Fig. 8). However for the slower waves, such as the Rayleigh waves, the presence of the cavity further amplifies the signal. Indeed, it appears that it is the presence of the cavity that mostly determines the dynamic behaviour of the full system at this line of receivers. Therefore, the influence of the surface deformation diminishes with the presence of this cavity for receivers placed along line 2. This behaviour was expected, given the greater dimension of the cavity relative to the size of the surface deformation, and the position of the line of receivers.

As we move to a lower apparent wave velocity, the time responses illustrate the importance of the Rayleigh waves, placing pulses with higher amplitude at receivers closer to the cavity surface, located at the medium depth of the second line of receivers (see Fig. 12b). These guided modes are mostly polarized along the direction normal to the cavity surface. So, the tangential component is less important. The vertical displacement, not displayed, confirms that the main field comes from Rayleigh waves that travel along the surface. We can conclude that the presence of the cavity itself dominates the behaviour of the wave field at these receivers.

(a)

Flat surface

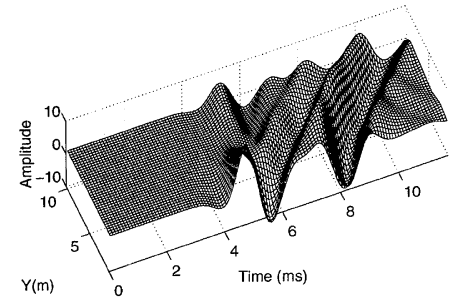

(b)

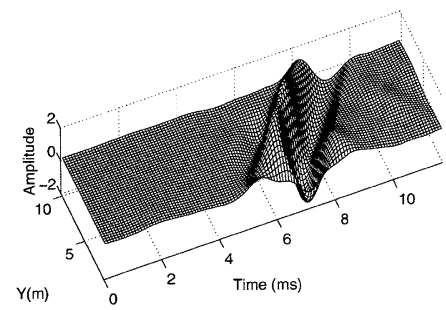

Smooth ridge
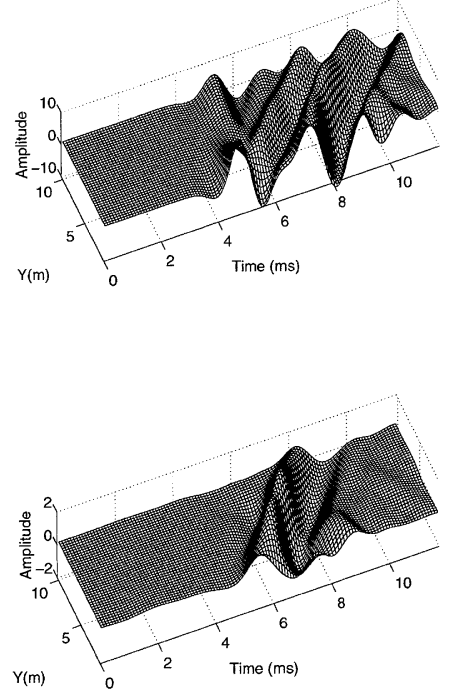

Smooth canyon
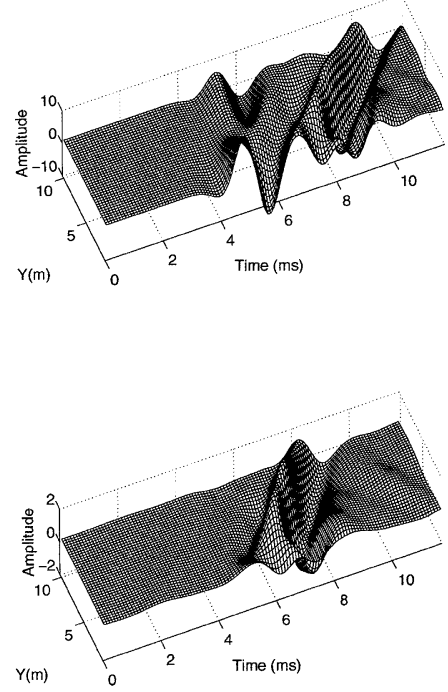

Fig. 12. Total horizontal displacement time responses recorded at the receivers placed along line 2 when a cylindrical circular cavity of $R=3 \mathrm{~m}$ is present: (a) Infinite $c$ and (b) $c=4208 \mathrm{~m} / \mathrm{s}$. 
Flat surface

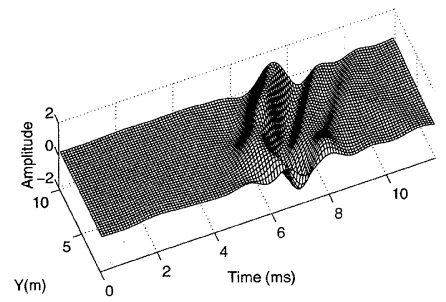

Smooth ridge

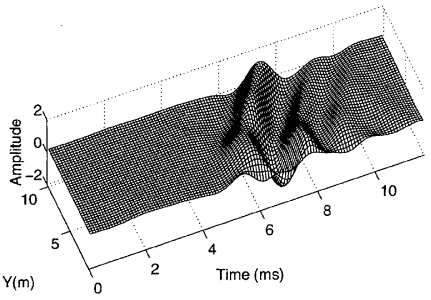

Smooth canyon

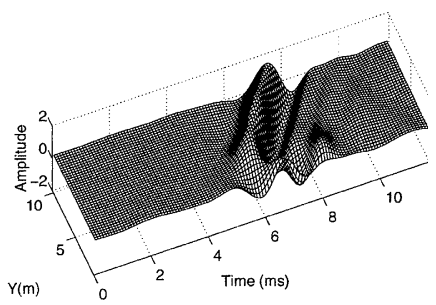

Fig. 13. Total horizontal displacement time responses recorded at the receivers placed along line 1 when a cylindrical circular cavity of $R=2 \mathrm{~m}$ is present for $c=4208 \mathrm{~m} / \mathrm{s}$.

Fig. 13 depicts the total horizontal time displacements when the cavity assumes a radius of $R=2 \mathrm{~m}$, for the receivers placed at line 1 . Analysis of the results reveals features similar to the ones observed when the radius of the cavity is $R=3 \mathrm{~m}$. However, it can easily be seen that the amplitude of the $S$ and Rayleigh waves decreases when we move to an inclusion with a radius of $R=2 \mathrm{~m}$ (see Figs. $9 \mathrm{~b}$ and 13). The loss of importance of the cavity can also be observed from the vertical time displacement results (not illustrated), with smaller but manifest multiple reflections between the topographical surface and the surface of the cavity. The calculated $z$ displacement field shows a similar tendency (not illustrated).

The disturbance caused by the presence of the cavity in the incident wave field, noticed as a shadow effect, is less apparent for a cavity with a lower radius at the second line of receivers. Fig. 14 illustrates this behaviour by displaying the horizontal displacements (see also Fig. 12), where higher amplitudes of the response occur at receivers placed outside the shadow zone.

It can also be observed that the importance of the amplitude of the guided waves is reduced when the radius of the cavity changes from $R=3 \mathrm{~m}$ to $R=2 \mathrm{~m}$ (see Figs. 12 and 14). The increased distance from the receivers to the cavity and its smaller size explain this behaviour, a tendency that is followed by the vertical and $z$ displacements (not illustrated).

From an engineering point of view, it is useful to know how an explosion may affect a tunnel such as subway or a railway tunnel. The answer to this question would require a new series of studies. However, the

(a)

Flat surface

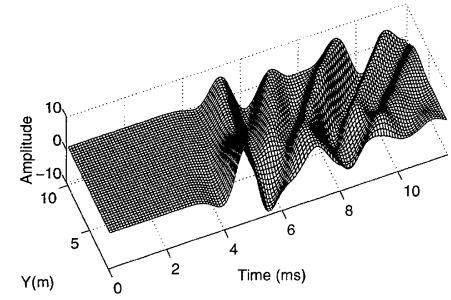

(b)

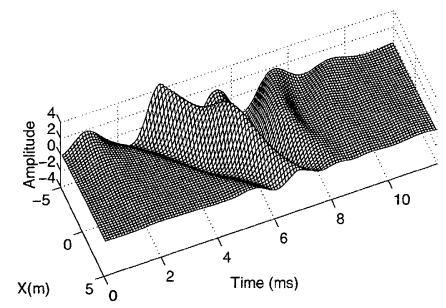

Smooth ridge
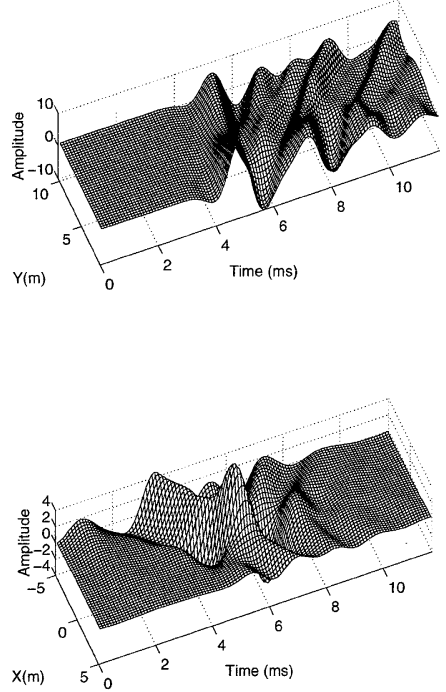

Smooth canyon
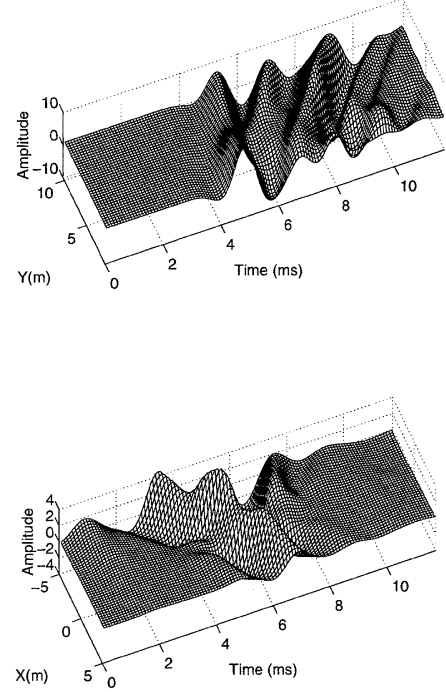

Fig. 14. Total horizontal displacement time responses recorded at the receivers placed along line 2 when a cylindrical circular cavity of $R=2 \mathrm{~m}$ is present: (a) Infinite $c$ and (b) $c=4208 \mathrm{~m} / \mathrm{s}$. 
results obtained in this paper indicate that amplification and de-amplification of the signals will occur in the close vicinity of the tunnel, generated by guided waves that will travel along its surface, giving rise to higher stress and deformation fields. This behaviour becomes increasingly important for tunnels placed closer to the surface of the ground, where the interaction with the surface waves produced will increase the complexity of the response and give rise to further amplification and de-amplifications of the signals.

\section{Conclusions}

The boundary element formulation developed was used to calculate the alteration of the 3D scattered field generated by a dilatational point load illuminating a halfspace, when the free surface is changed to model a smooth ridge or a valley with the presence or in the absence of a cylindrical circular cavity. The frequency and synthetic seismograms were first built when the elastic medium was free of any inclusion, following waves with different apparent wave velocities along the $z$-axis. The time responses appear complicated but were consistent with the predictions calculated by ray acoustics. Simulation analyses utilizing this idealized model were used by the authors to allow the recognition, identification, and physical interpretation of the variation of the wave field in the vicinity of these topographical deformations.

Larger wave field alterations, with the amplification and de-amplification of the signal in the time and frequency domains, occur when we move from the flat half-space and include the smooth ridge and canyon deformations. These originate within the concave parts of the surface deformations. As the apparent velocity decreases, the amplitude of the response decreases, indicating that the scattering energy is mainly concentrated in the vertical $z$ plane containing the dynamic source. It can further be observed that the canyon deformation creates a significant shadow after the surface deformation because the direct incident pulses are easily reflected onto the formation, leading to a zone where the responses decrease in amplitude.

Our simulations also included the evaluation of the response field in the presence of differently-sized cylindrical cavities, buried below the topographical surface deformation. The presence of an inclusion leads to a more complicated wave field displacement pattern, including additional reflections. However, the results continue to exhibit amplification and de-amplification differences close to the concave part of the surface deformations, for receivers just below the ground surface. The presence of the ridge allows higher order reflections to exist between the ground surface and the top of the inclusion, sustaining more energy there, for a longer time. The receivers placed in the vicinity of the canyon show smaller amplitudes than do the receivers that are further away. This phenomenon is explained in the light of the reflective power of the convex shape of the surface and of the cavity, which enables energy to be reflected to the sides, away from the central zone. It was found that the guided waves now have higher amplitudes than were observed for the homogeneous medium, because of the additional presence of guided waves that travel around the surface cavity.

The cavity gives rise to a so-called shadow effect at receivers behind the cavity when illuminated by the first incident $P$ body waves. However for the slower waves, such as the Rayleigh waves, the presence of the cavity further amplifies the signal. The influence of the surface deformation type diminishes at these receivers when a cavity is present. When the size of the inclusion decreases, the amplitude of the $S$ and Rayleigh waves decreases. Indeed, even though the presence of the cavity becomes less important, multiple reflections between the topographical surface and the surface of the cavity are still present.

\section{Appendix A}

\section{A.1. Definitions}

$\lambda, \mu \quad$ Lamé constants

$\rho \quad$ mass density

$\alpha=\sqrt{(\lambda+2 \mu) / \rho} P$ wave velocity

$\beta=\sqrt{\mu / \rho} \quad S$ wave velocity

$k_{p}=\omega / \alpha \quad k_{s}=\omega / \beta$

$k_{\alpha}=\sqrt{k_{p}^{2}-k_{z}^{2}} \quad k_{\beta}=\sqrt{k_{s}^{2}-k_{z}^{2}}$

$A=1 /\left(4 \mathrm{i} \rho \omega^{2}\right)$ amplitude

$\gamma_{i}=\partial r / \partial x_{i}=x_{i} / r \quad i=1,2$ direction cosines

$H_{n \alpha}=H_{n}^{(2)}\left(k_{\alpha} r\right) \quad H_{n \beta}=H_{n}^{(2)}\left(k_{\beta} r\right)$ Hankel functions

$B_{n}=k_{\beta}^{n} H_{n \beta}-k_{\alpha}^{n} H_{n \alpha} \quad B_{n}$ functions

\section{A.2. Green's functions}

$$
\begin{aligned}
& G_{x x}=A\left[k_{s}^{2} H_{0 \beta}-\frac{1}{r} B_{1}+\gamma_{x}^{2} B_{2}\right] \\
& G_{y y}=A\left[k_{s}^{2} H_{0 \beta}-\frac{1}{r} B_{1}+\gamma_{y}^{2} B_{2}\right] \\
& G_{z z}=A\left[k_{s}^{2} H_{0 \beta}-k_{z}^{2} B_{0}\right] \\
& G_{x y}=G_{y x}=\gamma_{x} \gamma_{y} A B_{2} \\
& G_{x z}=G_{z x}=\mathrm{i} k_{z} \gamma_{x} A B_{1} \\
& G_{y z}=G_{z y}=\mathrm{i} k_{z} \gamma_{y} A B_{1}
\end{aligned}
$$


A.3. Volumetric strain (super-index=direction of load)

$$
\begin{aligned}
\varepsilon_{\mathrm{Vol}}^{l} & =G_{x l, x}+G_{y l, y}+G_{z l, z} \\
& =A\left[\frac{\partial}{\partial x_{l}}\left(k_{s}^{2} H_{0 \beta}\right)+B_{0, x l x}+B_{0, y l y}+B_{0, z l z}\right] \\
& =A \frac{\partial}{\partial x_{l}}\left[k_{s}^{2} H_{0 \beta}+B_{0, x x}+B_{0, y y}+B_{0, z z}\right] \\
& =A \frac{\partial}{\partial x_{l}}\left[k_{s}^{2} H_{0 \beta}+\hat{\nabla}^{2} B_{0}\right]
\end{aligned}
$$

Note: $H_{0 \beta, l}=-k_{\beta} \gamma_{l} H_{l \beta}, H_{0 \beta, z}=-\mathrm{i} k_{z} H_{0 \beta}$

A.4. Strain components (tensor definition, not engineering)

$$
\begin{aligned}
\varepsilon_{i j}^{l} & =\frac{1}{2}\left(G_{i l, j}+G_{j l, i}\right) \\
& =\frac{1}{2} A\left(\delta_{i l} k_{s}^{2} H_{0 \beta, j}+\delta_{j l} k_{s}^{2} H_{0 \beta, i}+B_{0, i l j}+B_{0, j l i}\right) \\
& =\frac{1}{2} k_{s}^{2} A\left(\delta_{i l} H_{0 \beta, j}+\delta_{j l} H_{0 \beta, i}\right)+A B_{0, i j l}
\end{aligned}
$$

A.5. Strains for loads in the plane, $l=x, y$

$$
\varepsilon_{\mathrm{Vol}}^{l}=\gamma_{l} A\left(-k_{s}^{2} k_{\beta} H_{1 \beta}+k_{z}^{2} B_{1}+\frac{4}{r} B_{2}-B_{3}\right)
$$

$\varepsilon_{x x}^{l}=\gamma_{l} A\left(\left(\frac{2}{r} B_{2}-k_{s}^{2} k_{\beta} H_{1 \beta}\right) \delta_{x l}+\frac{1}{r} B_{2}-\gamma_{x}^{2} B_{3}\right)$

$\varepsilon_{y y}^{l}=\gamma_{l} A\left(\left(\frac{2}{r} B_{2}-k_{s}^{2} k_{\beta} H_{1 \beta}\right) \delta_{y l}+\frac{1}{r} B_{2}-\gamma_{y}^{2} B_{3}\right)$

$\varepsilon_{z z}^{l}=\gamma_{l} k_{z}^{2} A B_{1}$

$\varepsilon_{x y}^{l}=A\left(\left(\frac{1}{r} B_{2}-\frac{1}{2} k_{s}^{2} k_{\beta} H_{1 \beta}\right)\left(\delta_{x l} \gamma_{y}+\delta_{y l} \gamma_{x}\right)-\gamma_{x} \gamma_{y} \gamma_{l} B_{3}\right)$

$\varepsilon_{x z}^{l}=\mathrm{i} k_{z} A\left(\left(\frac{1}{r} B_{1}-\frac{1}{2} k_{s}^{2} H_{0 \beta}\right) \delta_{x l}-\gamma_{x} \gamma_{l} B_{2}\right)$

$$
\varepsilon_{y z}^{l}=\mathrm{i} k_{z} A\left(\left(\frac{1}{r} B_{1}-\frac{1}{2} k_{s}^{2} H_{0 \beta}\right) \delta_{y l}-\gamma_{y} \gamma_{l} B_{2}\right)
$$

A.6. Strain for axial loads, $l=z$

$$
\varepsilon_{\mathrm{Vol}}^{z}=\mathrm{i} k_{z} A\left(-k_{s}^{2} H_{0 \beta}+k_{z}^{2} B_{0}+\frac{2}{r} B_{1}-B_{2}\right)
$$

$$
\begin{aligned}
& \varepsilon_{x x}^{z}=\mathrm{i} k_{z} A\left(\frac{1}{r} B_{1}-\gamma_{x}^{2} B_{2}\right) \\
& \varepsilon_{y y}^{z}=\mathrm{i} k_{z} A\left(\frac{1}{r} B_{1}-\gamma_{y}^{2} B_{2}\right) \\
& \varepsilon_{z z}^{z}=\mathrm{i} k_{z} A\left(-k_{s}^{2} H_{0 \beta}+k_{z}^{2} B_{0}\right) \\
& \varepsilon_{x y}^{z}=-\mathrm{i} k_{z} \gamma_{x} \gamma_{y} A B_{2}
\end{aligned}
$$

$\varepsilon_{x z}^{z}=\gamma_{x} A\left(-\frac{1}{2} k_{s}^{2} k_{\beta} H_{1 \beta}+k_{z}^{2} B_{1}\right)$

$\varepsilon_{y z}^{z}=\gamma_{y} A\left(-\frac{1}{2} k_{s}^{2} k_{\beta} H_{1 \beta}+k_{z}^{2} B_{1}\right)$

\section{A.7. Stresses}

$\tau_{i j}^{l}=\lambda \varepsilon_{\mathrm{Vol}}^{l} \delta_{i j}+2 \mu \varepsilon_{i j}^{l}$

\section{References}

[1] Davis LL, West LR. Observed effects of topography on ground motion. Bull Seism Soc Am 1973;63:283-98.

[2] Griffiths DW, Bollinger GA. The effect of Appalachian mountain topography on seismic waves. Bull Seism Soc Am 1979;69:1081-105.

[3] Bard PY, Tucker ET. Underground and the ridge site effects: a comparison of observation and theory. Bull Seism Soc Am 1985;75:905-22.

[4] Trifunac MD. Surface motion of a semi-cylindrical alluvial valley for incident plane SH waves. Bull Seism Soc Am 1971;61:1755-70.

[5] Trifunac MD. Scattering of plane $\mathrm{SH}$ waves by a semicylindrical canyon. Int J Earthquake Engng Struct Dyn 1973;1:267-81.

[6] Wong HL, Trifunac MD. Surface motion of semi-elliptical alluvial valley for incident plane $\mathrm{SH}$-waves. Bull Seism Soc Am 1974;64:1389-403.

[7] Lee VW, Cao H. Diffraction of SV waves by circular canyons of various depths. J Engng Mech, ASCE 1989;115:2035-56.

[8] Todorovska MI, Lee VW. A note on response of shallow circular valleys to Rayleigh waves: analytical approach. Earthquake Engng Vib 1990;10:21-34.

[9] Todorovska MI, Lee VW. Surface motion of circular valleys of variable depth for incident plane $\mathrm{SH}$ waves. Soil Dyn Earthquake Engng 1991;10:192-200.

[10] Lee VW. On deformations near circular underground cavity subjected to incident plane SH waves. Symp Appl Comp Methods Engng, University of Southern California, Los Angeles, 1977. p. 951-61. 
[11] Datta SK, Shah AH. Scattering of SH-waves by embedded cavities. Wave Motion 1982;4:265-83.

[12] Lee VW. Three-dimensional diffraction of elastic waves by a spherical cavity in an elastic half-space. 1: Closedform solutions. Soil Dyn Earthquake Engng 1988;7:14961.

[13] Lee VW, Karl JA. Diffraction of SV waves by underground circular cylindrical cavities. Soil Dyn Earthquake Engng 1992;11:445-56.

[14] Sanchez-Sesma FJ. Diffraction of elastic waves by three dimensional surface irregularities. Bull Seism Soc Am 1983;73:1621-36.

[15] Moeen-Vaziri N, Trifunac MD. Scattering and diffraction of plane P and SV waves by two-dimensional inhomogeneities: Part II. Soil Dyn Earthquake Engng 1988;7:189200.

[16] Lee VW, Wu X. Application of the weighted residual method to diffraction by 2-D canyons of arbitrary shape: II. Incident P, SV and Rayleigh waves. Soil Dyn Earthquake Engng 1994;13:365-75.

[17] Waas G. Linear two-dimensional analysis of soil dynamics problems in semi-infinite layered media. $\mathrm{PhD}$ dissertation. University of California, Berkeley, CA, 1972.

[18] Lysmer J, Udaka T, Seed HB, Hwang R. LUSH- A computer program for complex response analysis of soilstructure systems. Report no. EERC 74-4. Earthquake Engineering Research Center. University of California, Berkeley, CA, 1974.

[19] Kausel E. Forced vibrations of circular foundations in layered media. MIT Research Report 70-3. Department of Civil Engineering. Massachusetts Institute of Technology. Cambridge, MA, 1974.

[20] Wong HL, Jennings PC. Effect of canyon topographies on strong ground motion. Bull Seism Soc Am 1975;65:123957.

[21] Zhang L, Chopra AK. Three dimensional analyses of spatially varying ground motions around a uniform canyon in a homogeneous half-space. Int $\mathrm{J}$ Earthquake Eng Struct Dyn 1991;20:911-26.

[22] Sánchez-Sesma FJ, Rosenblueth E. Ground motion on alluvial valleys under incident plane $\mathrm{SH}$ waves. Int J Earthquake Engng Struct Dyn 1979;7:441-50.

[23] Wong HL. Effect of surface topography on the diffraction of P, SV, and Rayleigh waves. Bull Seism Soc Am 1982;72:1167-83.

[24] Luco JE, Wong HL, De Barros FCP. Three-Dimensional response of a cylindrical canyon in a layered half-space. Int J Earthquake Eng Struct Dyn 1990;19:799-817.

[25] Sánchez-Sesma FJ, Campillo N. Diffraction of P, SV, and Rayleigh waves by topographical features: a boundary integral formulation. Bull Seism Soc Am 1991;81:2234 53.

[26] Sánchez-Sesma FJ, Campillo N. Topographical effects for incident $\mathrm{P}, \mathrm{SV}$, and Rayleigh waves. Tectonophysics 1993;218:113-25.
[27] Bouchon M. A simple, complete numerical solution to the problem of diffraction of SH waves by an irregular surface. J Acoust Soc Am 1985;77:1-5.

[28] Kawase H. Time-domain response of a semicircular canyon for incident SV, P and Rayleigh waves calculated by the discrete wavenumber boundary element method. Bull Seism Soc Am 1988;78:1415-37.

[29] Pei D, Papageorgiou AS. Three-dimensional response of an infinitely long cylindrical canyon to obliquely incident seismic waves (abstract). Seism Res Lett 1993;64:27.

[30] Ohtsuki A, Harumi K. Effect of topography and subsurface inhomogeneities on seismic SV waves. Int J Earthquake Engng Struct Dyn 1983;11:441-62.

[31] Shah AH, Wong KC, Datta SK. Diffraction of plane SH waves in a half-space. Int J Earthquake Engng Struct Dyn 1982;10:519-28.

[32] Sánchez-Sesma FJ. Site Effects on Strong Ground Motion. Soil Dyn Earthquake Engng 1987;6:124-32.

[33] Geli LL, Bard PY, Jullien B. The effect of topography on earthquake ground motion: a review and new results. Bull Seism Soc Am 1988;78:42-63.

[34] Stamos AA, Beskos DE. A 3-D seismic response analysis of long lined tunnels in half-space. Soil Dyn Earthquake Engng 1996;15:111-8.

[35] Pedersen HA, Sánchez-Sesma FJ, Campillo M. Threedimensional Scattering by two-dimensional topographies. Bull Seism Soc Am 1994;84:1169-83.

[36] Bouchon M, Aki K. Discrete wave-number representation of seismic-source wave field. Bull Seism Soc Am 1977;67:259-77.

[37] Phinney RA. Theoretical calculation of the spectrum of first arrivals in layered elastic mediums. J Geophys Res 1965;70:5107-23.

[38] Manolis GD, Beskos DE. Boundary Element Methods in Elastodynamics. London: Unwin Hyman (sold to Chapman and Hall); 1988.

[39] Banerjee PK. The boundary element methods in engineering. New York: McGraw-Hill; 1994.

[40] Brebbia CA, Telles JCF, Wrobel LC. Boundary Elements Techniques. Berlin: Springer; 1984.

[41] Tadeu AJB, Santos PFA, Kausel E. Closed-form integration of singular terms for constant, linear and quadratic boundary elements-Part I: SH wave propagation. EABE Engng Anal Boundary Elem 1999;23(8):671-81.

[42] Tadeu AJB, Santos PFA, Kausel E. Closed-form integration of singular terms for constant, linear and quadratic boundary elements-Part II: SV-P wave propagation. EABE - Engng Anal Boundary Elem 1999;23(9):757-68.

[43] Tadeu AJB, António JMP. Use of constant, linear and quadratic boundary elements in $3 \mathrm{D}$ wave diffraction analysis. EABE - Engng Anal Boundary Elem 2000; 24(2):131-44.

[44] Kausel E, Roesset JM. Frequency domain analysis of undamped systems. J Engng Mech, ASCE 1992;118(4): 721-34. 